\title{
Tecnologias de comunicação, entretenimento e competências cognitivas na cibercultura*
}

\begin{abstract}
RESUMO
As mídias digitais têm revolucionado a indústria de entretenimento. $\mathrm{O}$ interesse nessas mudanças advém do fato de que os produtos de divertimento contemporâneos parecem demandar não apenas atividades mentais, mas a ação do corpo e das formas cognitivas que não podem ser reduzidas às habilidades conteudísticas pelas quais julgamos a cultura de massa tradicional. Por se tratar de habilidades que requerem capacitação em diversas áreas, decidimos denominá-las competências cognitivas. O objetivo do presente texto é investigar quais competências cognitivas estariam sendo requeridas e estimuladas nas práticas comunicativas do usuário dos produtos modernos de entretenimento. Uma investigação preliminar nos encoraja a encaminhar a hipótese de que tais competências seriam sensoriais, lógicas, criativas, sociais e cibertextuais.
\end{abstract}

\section{PALAVRAS-CHAVE}

cibercultura

competências cognitivas

entretenimento

\begin{abstract}
The entertainment industry has been revolutionized by the digital midia. We are concerned with these changes because the current entertainment production seems to demand not only mental activities, but also the use of the body and of cognitive forms, irreducible to the content ordinarily used to judge traditional mass culture. How these abilities demand capacitating in many areas, we decided to call them cognitive competencies. The current article aims to investigate which cognitive competencies would be required and stimulated in the communication practices applied by the user to enjoy contemporary entertainment production. A preliminary investigation stimulate us to supose that these practices would be the following: sensorial, logical, creative, social and cybertextual.
\end{abstract}

\section{KEY WORDS}

cyberculture

cognitive competencies

entertainment

\section{Fátima Régis}

Professora do Programa de Pós-Graduação em Comunicação - UERJ/RJ/BR fregis@ueri.br
O que as crianças aprendem e a maneira como aprendem, depende, em grande parte, dos objetos que são colocados em suas mãos.Friedrich Froebel - educador alemão e criador do Kindergarten

A recombinação de formas, estilos, códigos e linguagens é uma prática antiga na cultura ocidental. Lev Manovich explica que "a maioria das culturas se desenvolveu tomando emprestado e retrabalhando formas e estilos de outras culturas; (...) A Roma Antiga remixou a Grécia Antiga, o Renascimento remixou a Antiguidade;"'1 (2005). Bolter e Grusin (1998, p. 36) afirmam que, já no século $\mathrm{XV}$, os pintores holandeses costumavam incorporar em suas obras espelhos, pinturas, mapas, gráficos, textos e outros elementos.

Os meios de comunicação não fogem à regra. Compartilham linguagens desde sua origem. O cinema incorporou técnicas e linguagens da fotografia e do teatro. $\mathrm{O}$ formato das histórias em quadrinhos foi inspirado na literatura e no cinema e assim por diante. Mas, a diferença de suporte material de cada mídia, "papel para o texto, película química para a fotografia e cinema, fita magnética para o som e o vídeo" (Santaella, 2003, p. 83), limitava uma imbricação mais completa entre mídias.

Com a característica das tecnologias de informação de transpor para a base digital meios e linguagens provenientes dos diversos tipos de mídia - como texto, ilustrações, fotos, sons, músicas, animações e vídeos - temos observado uma verdadeira revolução nos processos de gravação, produção, armazenamento e distribuição dos produtos midiáticos.

Esse processo tem sido muito discutido no âmbito das pesquisas em comunicação. Lucia Santaella (2003, p. 84) se refere a ele como convergência das mídias. Manovich o denomina remixabilidade: "o processo transformativo por meio do qual os meios e as informações que organizamos e compartilhamos podem ser recombinados e construídos de modo a criar novas formas, conceitos, idéias, mashups e serviços" ${ }^{2}$ (2005). Bolter e Grusin (1998) analisam essa convergência das mídias, indicando três processos por meio dos quais elas se concretizam: imediação, hipermediação e remediação. Para os autores, a remediação não foi inventada pelas mídias digitais, mas é característica definidora de tais meios.

O fato é que essa convergência das mídias, remixabilidade ou remediação, tem revolucionado a indústria de entretenimento contemporâneo: talentos artísticos são descobertos na internet; o download de seriados de TV incentiva seu ibope oficial; a avaliação de um livro por leitores alavanca a sua venda.

O interesse nessas mudanças advém do fato de que 
elas parecem demandar não apenas atividades mentais, mas a ação do corpo e de formas cognitivas que não se reduzem às habilidades representacionais pelas quais, tradicionalmente, julgamos a cultura do entretenimento.

O objetivo do presente texto é investigar, de forma sucinta e exploratória, quais competências cognitivas estariam sendo requeridas e estimuladas nas práticas comunicativas do usuário dos produtos de entretenimento contemporâneos.

Breve mapeamento sobre as competências cognitivas na cibercultura

As recombinações de textos, ilustrações, fotos, sons, músicas, animações e vídeos, inerentes aos processos de remediação, desafiam aspectos cognitivos como atenção, percepção e criatividade. Além dessas remediações, parece-nos que há dois outros fatores que desafiam os aspectos cognitivos das práticas de comunicação contemporâneas.

O primeiro se refere ao surgimento crescente de novas interfaces e equipamentos (Ipods, Iphones, Palm Tops, celulares com tecnologia $W A P$, equipamentos de simulação e de realidade virtual) que além de tornarem-se suportes para tais recombinações, exigem um refinamento das habilidades visuais, táteis e sonoras (visualização em telas minúsculas e divididas; manuseio de diversos tipos de joysticks e aparelhos de controles remotos; compreensão de novas interfaces e softwares; habilidades táteis finas para manuseio e digitalização em aparelhos pequenos, entre outras).

O segundo fator diz respeito aos recursos de comunicação em rede e de comunicação móvel que favorecem a produção, troca e compartilhamento de produtos e informações, incrementando o surgimento de redes sociais, comunidades virtuais, sites de relacionamento que requerem perspicácia no trato social e emocional.

Georg Simmel (1987), Walter Benjamin (1994), Jonathan Crary $(1992,2001)$ são alguns pesquisadores que analisam o modo que a intensidade e a diversidade dos estímulos das metrópoles modernas influenciaram a experiência subjetiva de seus habitantes.

Walter Benjamin destaca que a fragmentação visual e a montagem rápida do cinema traduzem a experiência de choque e intensidade dos estímulos da vida moderna:

O filme serve para exercitar o homem nas novas percepções e reações exigidas por um aparelho técnico cujo papel cresce cada vez mais em sua vida cotidiana. Fazer do gigantesco aparelho técnico do nosso tempo o objeto das inervações humanas - é essa a tarefa histórica cuja realização dá ao cinema o seu verdadeiro sentido (BENJAMIN, 1994, p. 174).

Para Benjamin, o desenvolvimento da gramática cinematográfica - com suas características de fragmentação, montagem rápida e síntese do movimento - teve uma dupla função pedagógica: 1) capacitar o espectador para pari passu aprender a linguagem do cinema; 2) capacitar o cidadão para os estímulos e mudanças introduzidas pela vida na cidade.
De fato, a gramática cinematográfica, desenvolvida ao longo das três primeiras décadas do cinema, capacitou o cidadão moderno. Segundo a lenda, os espectadores da primeira sessão, ao ver a imagem de L'Arrivée d'un train à La Ciotat, confundiram-na com a realidade e saíram correndo da sala. Seus órgãos sensoriais e perceptivos nunca haviam sido submetidos à experiência de assistir a uma imagem em movimento que representa a realidade. Portanto, não souberam diferenciar entre a imagem do trem representada na tela e a realidade. Quarenta anos depois, esses espectadores e/ou seus filhos assistiram a filmes como E o Vento levou (1939) e Cidadão Kane (1941) completamente adaptados aos efeitos especiais e aos recursos lingüísticos mais sofisticados.

Assim como o cinema operou enquanto ferramenta de capacitação cognitiva para a experiência urbana, hoje os produtos de entretenimento baseados nas tecnologias digitais parecem fornecer um tipo semelhante de habilitação.

Ao discutir a revolução do mercado cultural contemporâneo, diversos teóricos oferecem pistas sobre essa capacitação cognitiva das tecnologias de informação e de comunicação. Buscamos, a seguir, destacar (em negrito) algumas das competências que estariam sendo exigidas/estimuladas nos indivíduos e o modo como interferem nos processos comunicativos contemporâneos.

Lúcia Santaella destaca que a partir da década de 1980 apareceram dispositivos que potencializaram as cópias, como videocassetes e fotocopiadoras, gerando uma cultura do disponível e do transitório: surgiram videogames, videoclips e TV a cabo.

Esses dispositivos tecnológicos e as linguagens criadas para circularem neles propiciaram a escolha e o consumo individualizados em oposição ao massivo, fornecendo o treinamento adequado para buscarmos as informações e os entretenimentos desejados com a chegada dos meios digitais (SANTAELLA, 2003, pp.12-17 - grifos nossos).

Steven Johnson diagnostica que avaliamos os produtos da cultura de massa e do entretenimento popular por sua capacidade de nos proporcionar conteúdos eruditos e lições morais. Johnson propõe o deslocamento da ênfase no conteúdo para outros aspectos que aguçam nossas habilidades mentais, sem pertencerem ao campo da hermenêutica: "Acho que há outra forma de avaliar a virtude social da cultura popular, uma que analise a mídia como uma espécie de treinamento cognitivo e não como uma série de lições de vida" (2005, p. 12 - grifos nossos), conclui.. Johnson sugere três fatores por meio dos quais a Internet desafia nossas habilidades mentais: "pela virtude de ser participativa; por forçar os usuários a aprenderem novas interfaces e novos softwares; e, pela criação de novos canais para a interação social". (2005, p. 93 - grifos nossos)

Chris Anderson, em seu já clássico A Cauda Longa, analisa a evolução do mercado cultural desde antes da revolução industrial até a recente revolução do formato 
digital. Anderson argumenta que com a disponibilidade de toda sorte de produto e gênero na rede, os consumidores não precisam ficar restritos aos hits e podem se dispersar. Na medida em que os consumidores se dispersam, o mercado se fragmenta em inúmeros nichos, formando grupos por afinidades e interesses comuns, diferentemente da era pré-industrial separada pela geografia.

Segundo Anderson, as redes de compartilhamento de arquivo (peer-to-peer) reúnem 10 milhões de usuários compartilhando músicas e filmes todos os dias. Essas pessoas pararam de comprar $\mathrm{CD}$ e perderam o gosto pelos grandes hits, elas querem explorar novidades. Além de divulgar e distribuir as produções de artistas independentes, a internet favorece a criação de novos modelos de "arte", como os mashups (tocar a faixa de um artista sobre outra) e os spoofs (criações sobre vídeos) pelo usuário comum.

Umberto Eco denomina intertextualidade uma característica muito encontrada nos produtos da cultura de massa tradicional que tem sido levada ao ápice pelas mídias digitais. Intertextualidade é a capacidade de o produto de uma mídia (livro, filme, videogame etc) citar direta ou indiretamente, por meio de repetição, paráfrase ou outro recurso lingüístico, uma cena de filme, um trecho de obra literária, uma frase musical. Nas palavras do autor é um processo em que um texto "cita, de modo mais ou menos explícito, uma cadência, um episódio, um modo de narrar que imita o texto de outrem" (1989, p. 125). Conhecer previamente os textos citados ou homenageados é um desafio proporcionado pela obra para que o indivíduo consiga ter acesso às diversas possibilidades e elementos de decodificação da obra. A esse repertório prévio Eco denomina enciclopédia intertextual. Além do repertório intertextual, atenção e percepção acurada para capturar essas mensagens escondidas são outras habilidades requeridas ao público.

Já o teórico norueguês Espen Aarseth cunhou dois termos para estudar as especificidades dos videogames: literatura ergódica e cibertextos. A idéia de literatura ergódica vem de Ergodic, do grego ergon (trabalho) e hodos (caminho). Refere-se a um tipo de texto que demanda do "usuário" um trabalho físico, corporal, um esforço nãotrivial, distinto do esforço que seria, por exemplo, a interpretação de um texto ou a movimentação dos olhos pela página no ato da leitura tradicional.

Os exemplos de literatura ergódica são o I-Ching, os MUD (Multi-User Dungeons) ${ }^{3}$, algumas obras de vanguarda como The Unfortunates (1969) de B. S. Johnson e Rayuela, de Julio Cortazar e, evidentemente, os jogos de computador. A esses textos que demandam um desempenho corporal por parte do "leitor", Aarseth denomina cibertextos. Aarseth entende os cibertextos como máquinas literárias e seus leitores como jogadores que devem não apenas "ler", mas "explorar" o ambiente, perder-se, descobrir bônus e caminhos secretos.

É interessante comparar a descrição das habilidades cibertextuais de Aarseth com duas habilidades que Johnson (2005) descreve como sendo inerentes aos jogos: sondagem e investigação telescópica. Johnson explica que grande parte dos videogames nos coloca diante de situações nas quais é preciso tomar decisões. Defende que aprender a tomar a decisão correta tem a ver com "aprender como pensar", o que atribui aos jogos a capacidade de desenvolver habilidades "intelectuais tradicionais", como resolução de problemas, tomada de decisão e lógica.

Para Johnson, além da destreza manual ou visual, os jogos estimulam duas habilidades intelectuais fundamentais: a sondagem e a investigação telescópica.

Ao contrário de jogos tradicionais como o xadrez, no mundo do videogame as regras raramente são estabelecidas na íntegra antes do início do jogo. Freqüentemente a meta do jogo e as técnicas para alcançá-la não são conhecidas previamente: "tornam-se evidentes por meio da exploração do mundo" (Johnson, 2005, p. 35). Continua o autor: "Você tem que sondar as profundezas lógicas do jogo para entendê-lo e, como na maioria das expedições investigativas, você obtém resultados por meio de tentativa e erro, tropeçando nas coisas, seguindo intuições" (2005, p. 35 - grifos nossos).

A outra habilidade intelectual é a investigação telescópica. A partir da década de 1990, os videogames passaram a oferecer um número maior de objetivos que precisam ser mentalmente organizados, de modo aninhado e hierárquico. Para o autor: "Chamo o trabalho mental de gerenciar simultaneamente todos esses objetivos de 'investigação telescópica' devido ao modo como eles se aninham um dentro do outro como um telescópio desmontado" (2005, p. 43). Ou seja, a consecução de um objetivo implica outro que implica outro e assim por diante. $\mathrm{O}$ jogador precisa não apenas ter mentalmente organizados todos esses objetivos, mas também, direcionar sua percepção visual e seus reflexos sensório-motores a eles.

A breve exposição acima permite perceber que praticamente há um consenso em relação ao fato de que a indústria do entretenimento vem se transformando a "olhos vistos". Mas, parece haver uma lacuna sobre qual seria exatamente a natureza dessa mudança. Por exemplo, se na Modernidade o desenvolvimento da atenção foi fundamental para a concentração e a capacidade seletiva do indivíduo (Cf. Crary, 2001), hoje parece que, além da atenção e percepção seletivas extremamente aguçadas para realizar tarefas como a busca da informação desejada e a exploração no mundo dos jogos, o indivíduo precisa de uma dose de desatenção (déficit de atenção) para captar as informações fragmentadas no meio ambiente e realizar múltiplas tarefas simultaneamente.

Apesar de encontrarmos em vários autores o diagnóstico dessas mudanças, observamos uma lacuna referente a um mapeamento mais completo e acurado sobre quais são as competências envolvidas e como afetam as práticas comunicativas contemporâneas.

Um fator instigante é que as habilidades descritas 
pelos autores citados pertencem a um amplo repertório cognitivo, envolvendo capacidades sensoriais, perceptivas, lingüísticas, criativas e sociais, além de atividades relacionadas às formas tradicionais de inteligência, tais como a lógica, a resolução de problemas, a análise, o reconhecimento de padrões e a tomada de decisão.

Os produtos de entretenimento atuais parecem demandar além das atividades mentais, a ação do corpo e de formas cognitivas que não podem ser reduzidas às habilidades representacionais e conteudísticas pelas quais costumamos julgar a cultura de massa. Por se tratar de competências que requerem capacitação em diversas áreas decidimos denominá-las competências cognitivas. Escolhemos o termo cognição porque, de acordo com as ciências cognitivas, o conceito de cognição abrange todas as atividades e processos operados pela mente (Cf. LAKOFF,1999; CLARK, 2001). Por sua vez, a mente seria o resultado de um longo processo evolutivo que envolve as relações entre corpo e cérebro e suas interações com o ambiente (Dennett, 1996; Lakoff, Johnson, 1999). Para pensadores como Daniel Dennett, Georg Lakoff e Mark Johnson, os processos mentais envolvem não apenas as habilidades tradicionalmente classificadas como mentais (lógicas e racionais), mas todas as habilidades humanas, incluindo as sensório-motoras, perceptivas, emocionais e sociais.

Nas ciências cognitivas, o termo cognitivo é usado para qualquer tipo de operação ou estrutura mental. (...) Dessa forma, o processo visual classifica-se como cognitivo, assim como o processo auditivo. (...) Memória e atenção classificam-se como cognitivas. Todos os aspectos do pensamento e da linguagem, conscientes ou inconscientes, são assim cognitivos. (...) Imagens mentais, emoções e a concepção de operações motoras também são estudadas sob uma perspectiva cognitiva.

(...)

Porque nossos sistemas conceituais e nossa razão surgem de nossos corpos, também usaremos o termo cognitivo para aspectos de nosso sistema sensório-motor que contribuem para nossas habilidades de conceituar e raciocinar $^{4}$ (1999, pp. 11-12).

Nesta acepção, processos cognitivos envolvem não apenas a linguagem simbólica e representacional, mas todo o tipo de sinais e estímulos sensoriais e perceptivos que permitem tanto a formulação de códigos e linguagens atuantes em um regime de representação, quanto de outros processos de percepção, intensidades e afetuosidades que extrapolam o campo da linguagem, embora construam igualmente nossas práticas comunicativas e de sociabilidade.

Por competências cognitivas entendemos o conjunto de conhecimentos (saberes), habilidades (saber fazer) e atitudes (saber ser) necessárias para os usos, criações e recombinações de linguagens, interfaces e códigos pro- movidos pelas tecnologias digitais nas práticas de comunicação e de entretenimento contemporâneas.

\section{Competências Cognitivas na Cibercultura}

Para fins de categorização e possibilidade de criação de uma metodologia de pesquisa ${ }^{5}$, agrupamos as competências observadas em cinco categorias de análise: cibertextuais, sensoriais, lógicas, criativas e sociais.

As cinco categorias são o ponto de partida para o estudo das competências que estariam sendo requeridas e estimuladas nas práticas comunicativas do entretenimento contemporâneo. Essas categorias operam integradas entre si e algumas habilidades atuam nas suas interseções, mas para fins didáticos, optamos por enumerá-las separadamente:

A cibertextualidade é uma junção do conceito de cibertextos de Aarseth com a noção de intertextualidade de Eco. Compreende o conjunto de características das TIC que ao possibilitar a hibridação de meios, linguagens e textualidades, afeta a produção de textos, sua leitura e participação do leitor. Tal como Aarseth, acreditamos que as mídias digitais demandam um esforço não-trivial, envolvendo habilidades sensoriais e lógicas. A cibertextualidade refere-se também aos textos que citam intencionalmente outro produto cultural, estimulando a conexão de diferentes produtos culturais e os processos de leitura ergódica, produção e associação de conteúdo, diferenciados em relação à leitura linear.

A logicidade se refere ao estímulo às habilidades mentais tradicionalmente ligadas à lógica e resolução de problemas. A esta categoria pertencem as tarefas que aprimoram o caráter lógico, tais como: tomada de decisão, análise e reconhecimento de padrões. Neste item estão também as atividades associativas indispensáveis à formação da enciclopédia intertextual e de orientação espacial.

A criatividade se dedica às habilidades que estimulam a criação e participação nas atividades colaborativas na rede. Investiga o estímulo à intervenção nos produtos, por parte dos usuários, seja pela construção de obras inéditas ou pela criação por meio de mixagens, fanfictions, paródias, mashup e spoofs. Essa categoria envolve a construção social de conhecimento por meio dos recursos de blogs, redes P2P e redes de relacionamento que constituem a chamada Web 2.0 (Cf. O' reilly, 2005).

A sensorialidade examina como, ao reunir vários meios simultaneamente e combinar linguagens e textualidades distintas, os novos gadgets (aparelhos celulares, IPo$d s$, DVDs, capacetes de realidade virtual, plataformas de videogames) oferecem tecnologias que despertam diversos sentidos e desafiam as capacidades sensoriais, perceptivas, táteis, imersivas e de atenção (telas sensíveis ao toque, habilidades de visualização em telas minúsculas e divididas, manuseio de diversos tipos de joysticks e aparelhos de controles remotos; atenção e percepção seletivas para localizar a informação desejada).

A sociabilidade se refere ao modo como as tecnologias 
digitais, ao favorecerem a produção de conteúdo, incentivam o indivíduo a esquadrinhar as diversas mídias em busca da informação desejada e a engendrar um processo de colaboração entre indivíduos que se reúnem em comunidades virtuais, listas de discussão, blogs para buscar, produzir e partilhar informações adicionais sobre seus produtos culturais favoritos.

Essa "participação" dos espectadores se reflete na criação de uma rede de comentários da mídia, ou seja, o conjunto de meios de comunicação utilizados pelos usuários para trocar informações sobre seus produtos midiáticos preferidos. Essa rede não é invenção da cibercultura. Há décadas que filmes, seriados de televisão, livros e histórias em quadrinhos contam com cadernos culturais em jornais, revistas de entretenimento, documentários que comentam episódios, estruturas narrativas e motivação de personagens. Mas, o ápice da rede de comentários da mídia encontra seu suporte na internet, cujos blogs, sites, listas de discussão, softwares colaborativos, redes de relacionamento tornam-se recursos complementares de cognição dos programas, e nas mídias móveis (celulares, Ipods, etc.).

Essa rede de comentários da mídia é o que Bolter e Grusin chamam de remediação como mediação da mediação:

Cada ato de mediação depende de outros atos de mediação. Os Meios estão continuamente comentando, reproduzindo, e substituindo uns aos outros, e esse processo é fundamental para os meios. Os meios precisam uns dos outros de modo a funcionar como meios (BOLDER, GRUSIN, 1998, p. 54).

\section{Considerações finais}

Os estudos sobre o entretenimento midiático sempre foram alvo de discussões acaloradas no âmbito acadêmico sem que, no entanto, se chegasse a conclusões definitivas sobre o fenômeno. Tradicionalmente, os meios de comunicação e os produtos culturais por eles veiculados têm sido avaliados por seus conteúdos (Escola de Frankfurt, Sociedade do Espetáculo, Teoria Estruturalista). Ao enfatizar a capacidade de representação da sociedade e os valores ideológicos, essas abordagens avaliam os produtos de entretenimento como de padrão inferior e baixo nível cultural e estético.

Estudos recentes têm se dedicado a problematizar essa tendência. No cenário nacional, pesquisadores como Erick Felinto (2001) e Simone de Sá (2004) têm demonstrado que as leituras tradicionais, ao avaliar os produtos da cultura popular pelo viés do conteúdo, não abarcam sua complexidade. Felinto busca na teoria das materialidades, fundamentos para defender "a idéia de que a materialidade do meio de transmissão influencia e até certo ponto determina a estruturação da mensagem comunicacional" (Ciberlegenda, 2001). Sá destaca os suportes materiais e as relações que provocam na articulação corpo-tecnologia, privilegiando as dimensões sensoriais e cognitivas da cultura de massa.
A proposta aqui apresentada sobre as competências cognitivas que estariam sendo estimuladas pelos produtos de entretenimento na cibercultura é de caráter exploratório. Seu desenvolvimento buscará colaborar com o debate da área, aprofundando as discussões sobre: o estatuto do entretenimento na atualidade, a complexificação cognitiva dos produtos de entretenimento e as práticas comunicacionais contemporâneas mFAMEcos

\section{NOTAS}

* Este texto foi apresentado no II Simpósio Nacional da ABCiber realizado em novembro de 2008

1 Tradução livre para: "most human cultures developed by borrowing and reworking forms and styles from other cultures; (...) Ancient Rome remixed Ancient Greece; Renaissance remixed antiquity". (MANOVICH, 2005)

2 Tradução livre para "A transformative process in which the information and media we've organized and shared can be recombined and built on to create new forms, concepts, ideas, mashups and services". (MANOVICH,2005)

3 Jogos em que vários participantes podem jogar simultaneamente. Os MUD surgiram em 1980 e na medida em que foram de desenvolvendo passaram a permitir que os usuários construíssem suas próprias paisagens e objetos textuais.

4 In cognitive science, the term cognitive is used for any kind of mental operation or structure. (...) Thus, visual processing falls under the cognitive, as does auditory processing. (...) Memory and attention fall under the cognitive. All aspects of thought and language, conscious or unconscious, are thus cognitive. Mental imagery, emotions, and the conception of motor operations have also been studied from such a cognitive perspective. (...) Because our conceptual systems and our reason arise from our bodies, we also use the term cognitive for aspects of our sensorimotor system that contribute to our abilities to conceptualize and to reason.

5 As categorias aqui apresentadas são resultantes da pesquisa exploratória empreendida para a elaboração do projeto de pesquisa Tecnologias de Comunicação e Novas Habilidades Cognitivas na Cibercultura, financiado pela Faperj no triênio 2008-2011.

\section{REFERÊNCIAS}

AARSETH, E. Cybertext: perspectives on ergodic literature. Baltimore/London: The John Hopkins University Press, 1997.

ADORNO, T., HORKHEIMER, M. Dialética do esclareci- 
mento. Rio de Janeiro: Jorge Zahar Editor, 1985.

ANDERSON, C. The long tail. 2001. Disponível em: <http://www.wired.com/wired/archive/12.10/ tail.html>

BENJAMIN, W. “A obra de arte na era de sua reprodutibilidade técnica". In: Obras escolhidas: magia e técnica, arte e política: ensaios sobre a literatura e a história da cultura. 7 ed. São Paulo: Brasiliense, 1994.

BOLTER, J. D., GRUSIN, R. Remediation: understanding new media. Cambridge, Massachussets, 1998.

CLARK, A. Being there: putting brain, body and world together again. Londo: Bradford Book, 2001.

CLARK, A. Mindware: an introduction to the philosophy of cognitive science. New York/Oxford: Oxford University Press, 2001.

CRARY, J. Suspensions of perception: Attention, spectacle and modern culture. The MIT Press: October Books, 2001

CRARY, J. Techniques of the observer: On vision and Modernity of the 19th century. October Books, 1992.

DENNETT, D. Kinds of minds. New York: Basic Books, 1996.

ECO, U. "A Inovação no seriado". In: Sobre os espelhos e outros ensaios. Rio de Janeiro: Nova Fronteira, 1989.

FELINTO, E. Materialidades da comunicação: por um novo lugar da matéria na teoria da comunicação. Revista Eletrônica Ciberlegenda, no. 5, 2001.

GABLER, N. Vida, o filme. Como o entretenimento conquistou a realidade. São Paulo: Companhia das Letras, 1999.

GAUNTLETT, D. (org). Web.studies: Rewriting media studies for the digital age. London: Arnold, 2000.

JOHNSON, S. Surpreendente: a televisão e o videogame nos tornam mais inteligentes. Rio de Janeiro: Elsevier, 2005.

KOLLOCK, P. SMITH, M. (eds). Communities in cyberspace. London: Routledge, 1999.

LAKOFF, G. JOHNSON, M. Philosophy in the flesh. Nova York: Basic Books, 1999.

MANOVICH, L. Remixing and remixability. 2005. Disponível em <http://www.manovich.net/DOCS/ Remix_modular.doc $>$
O'REILLY, T. What is web 2.0. 2005. Disponível em: <http://www.oreillynet.com/pub/a/oreilly/tim/ news/2005/09/30/what-is-web-20.html>

RÉGIS, F. Da cultura de massa à cultura ciber: a complexificação da mídia e do entretenimento popular. INTERCOM, 2007, Santos, 29/08 a 02/09. Anais... VII Encontro dos Núcleos de Pesquisa em Comunicação - NP Tecnologias da Informação e da Comunicação.

RÉGIS, F. Tecnologias de comunicação e novas habilidades cognitivas na cibercultura. Projeto de Pesquisa aprovado pelo Programa Prociência. Rio de Janeiro: Faperj/ Uerj, 2008.

SÁ, S. P de. Por uma genealogia da noção de materialidade da comunicação. INTERCOM: NP Tecnologias da informação e da comunicação, 2004.

_. Quem media a cultura do shuffle? Cibercultura, mídias e cenas musicais. Porto Alegre: Revista Famecos/PUCRS, n. 15, jul. 2006.

SANTAELLA, L. Cultura e artes do pós-humano: da cultura das mídias à cibercultura. São Paulo: Editora Paulus, 2003.

SIMMEL, G. "A metrópole e a vida mental”. In: VELHO, G. (Org.). O fenômeno urbano. Rio de Janeiro: Ed. Guanabara, 1987.

VARELA, F. Conhecer: as ciências cognitivas, tendências e perspectivas. Lisboa: Instituto Piaget, s/d. 